\title{
GALECTINA-9 (GAL-9) ESTÁ PRESENTE NO SORO DE PACIENTES COM CÂNCER DE PÂNCREAS, MAS NÃO NO SOBRENADANTE DE LINHAGENS TUMORAIS PANCREÁTICAS.
}

\author{
L. B. TAVARES 1,2 , A. F. S. FILHO 1,2, K. M. VILAR 1,2, M. R. MARTINS4, M. G. R. \\ PITTA1,2,3, I. R. PITTA3 e M. J. B. M. RÊGO1,2,3
}
1 Universidade Federal de Pernambuco, Centro de Ciências Biológicas, Programa de Pós- graduação em Inovação Terapêutica
2 Universidade Federal de Pernambuco, Centro de Ciências Biológicas, Departamento de Bioquímica.
3 Universidade Federal de Pernambuco, Núcleo de Pesquisa em Inovação Terapêutica (NUPIT)
4 Hospital do Câncer de Pernambuco (HCP)
E-mail para contato: lucas.batista_tavares@hotmail.com

\begin{abstract}
RESUMO - O câncer de pâncreas está associado a um prognóstico ruim, diagnóstico tardio, e apresenta resistência aos tratamentos disponiveis atualmente. Galectinas participam de uma série de vias celulares que podem contribuir para o comportamento das células tumorais. Galectina-9 (Gal-9) atua na regulação da apoptose, interação celular, processo de invasão e angiogênese. Este estudo teve por objetivo avaliar os niveis de Gal-9 presentes no soro de pacientes acometidos pelo câncer de pâncreas e nos sobrenadantes de linhagens celulares representantes desta neoplasia. Estes foram mensurados por ELISA sanduiche. Todos os 29 pacientes com câncer de pâncreas apresentaram positividade para níveis séricos de Gal-9 (mediana $=7462)$, sendo observado nesses indivíduos um aumento significativo $(p<0,0001)$ nos valores dessa proteina circulante quando comparados a controles saudáveis (mediana=46.88). Já os níveis de Gal-9 presentes em sobrenadantes de linhagens tumorais pancreáticas Mia PaCa2 e PANC-1 ficaram abaixo do limite de detecção $(<46,875 \mathrm{pg} / \mathrm{ml}$.). Pacientes com câncer de pâncreas apresentam níveis séricos de Gal-9 alterados.

Palavras-chave: Glicobiologia. $\beta$-galactosídeos. Inovação Terapêutica.
\end{abstract}

\begin{abstract}
Pancreatic cancer is associated with a bad prognosis, late diagnosis, and resistance to available treatments. Galectins participate in various cell pathways that may contribute to the behavior of tumor cells. Galectin-9 (Gal-9) acts in the apoptosis regulation, cell interaction, invasion and angiogenesis processes. This study aimed to evaluate the expression of Gal-9 in pancreatic cancer. Serum levels of Gal- 9 in pancreatic tumor patients and pancreatic tumor cells lines supernatants exposed to hypoxia and nutritional were measured by sandwich ELISA. All 29 pancreatic cancer patients presented positive serum levels of Gal-9, with a significant increase $(p<0,0001)$ of this circulating protein when compared to healthy individuals. However, Gal-9 levels in supernatants of pancreatic tumor cells exposed to hypoxia and nutritional deprivation conditions for 24 and 48 hours were below the limit of detection $(<46,875$ $\left.\mathrm{pg} / \mathrm{ml}, R_{2}=0,8388\right)$. Patients with pancreatic cancer have altered serum levels of Gal-9.
\end{abstract}

Keywords: Glycobiology. $\beta$-galactoside. Therapeutic Innovation. 


\section{INTRODUÇÃO}

O câncer de pâncreas é a quarta principal causa de morte por câncer em países desenvolvidos, apresentando em cerca de 7\% dos casos uma taxa de sobrevivência geral de 5 anos (XU et al., 2014). Devido à dificuldade na identificação do tumor em estágios mais precoces, o câncer de pâncreas apresenta com frequência um prognóstico ruim, tendo possibilidade de ser realizada a ressecção cirúrgica em somente $15-20 \%$ dos casos (CID-ARREGUI; JUAREZ, 2015). Entre as neoplasias malignas que acometem o pâncreas, 80-90\% são representadas pelo tipo adenocarcinoma ductal pancreático, que por sua vez, é caracterizado pela intensa reação desmoplásica, com presença de fibroblastos, células inflamatórias e excessiva massa fibrótica, além do aumento significativo do fluido intersticial (WOLFGANG et al., 2013). Além disso, outra característica fundamental nesses tumores, é a presença de regiões hipóxicas que, assim como ocorre na maioria dos tumores sólidos, há uma diminuição dos níveis de oxigênio, que contribui para maior agressividade da doença (CÁRDENAS-RODRÍGUEZ et al., 2012). Isto acontece pela capacidade do microambiente desmoplásico e hipóxico de favorecerem a expressão de diversas proteínas com funções essenciais para $o$ desenvolvimento e a sobrevivência do tumor (LIU, Y.; DU, L., 2015).

Galectinas são proteínas da família das lectinas, com afinidade por $\beta$ galactosídeos, e estão envolvidas em diversas funções no desenvolvimento do câncer (KIM et al., 2013). A Galectina-9 (Gal-9) é uma glicoproteína que apresenta dois domínios de reconhecimento de carboidratos (LIU, F. T.; RABINOVICH, G. A., 2005). A mesma pode apresentar uma variedade de atividades biológicas no contexto tumoral: exercendo um papel regulador do ciclo celular e apoptose, contribuindo para a adesão, migração e metástase, além da fuga da resposta imunológica e influência na angiogênese (HEUSSCHEN, R.; GRIFFIOEN, A. W.; THIJSSEN, V. L., 2013).

\section{MATERIAIS E MÉTODOS}

2.1 RECRUTAMENTO DE PACIENTES - Foram incluídos 29 pacientes de ambos os sexos com idade entre 40 e 70 anos comprovadamente diagnosticado com câncer de pâncreas que estavam sendo acompanhados pelo setor de oncologia do Hospital do Câncer de Pernambuco (HCP) e ainda não tinham sido submetidos a nenhum tipo de tratamento. Estes, após assinarem o Termo de Consentimento Livre e Esclarecido (TCLE) se submeteram a coleta de sangue periférico através de punção venosa. Um grupo controle pareado por sexo e faixa etária, sendo formado por voluntários saudáveis sem diagnóstico de $\mathrm{CP}$ e sem histórico de $\mathrm{CP}$ familiar também foi incluído no estudo. O projeto possui aprovação pelo Certificado de Apresentação para Apreciação Ética (CAAE: 44002815.2.1001.5205).

2.2 PROCESSAMENTO DAS AMOSTRAS - Os tubos de coleta contendo cerca de 9 $\mathrm{mL}$ de sangue foram centrifugados nas condições de $2.500 \mathrm{rpm} / 10$ minutos, os soros depositados em eppendorfs e posteriormente armazenados em freezer $-80{ }^{\circ} \mathrm{C}$.

2.3 CULTIVO CELULAR - Duas linhagens celulares de carcinoma de pâncreas foram utilizadas nesse estudo. A célula MIA PaCa-2 foi mantida em cultura com meio DEMEM high glucose (Sigma-Aldrich) suplementado com 10\% de soro fetal bovino (SFB) acrescido de $2,5 \%$ de soro equino (Cultilab). A linhagem PANC-1 foi cultivada em meio DEMEM suplementado com $10 \%$ de SFB, HEPES $10 \mathrm{mM}$ (ácido N-2hidroxietilpiperazina-N-2-etanosulfonico), bicarbonato de sódio $24 \mathrm{mM}$, penicilina e estreptomicina $200 \mathrm{U} / \mathrm{mL}$ em estufa de atmosfera úmida a $37^{\circ} \mathrm{C}$, com $5 \%$ de $\mathrm{CO}_{2}$. 
2.4 DOSAGEM DE GALECTINA-9 - Níveis de galectina-9 presentes no soro de pacientes com câncer de pâncreas e no sobrenadante das linhagens Mia PaCa-2 e PANC1, foram quantificados por ELISA (Enzyme linked immuno sorbent assay) sanduíche, seguindo as informações recomendadas pelos fornecedores (R\&D Sistems). As avaliações foram feitas em duplicata, com limite de detecção do kit entre 46,8 pg/ml $6000 \mathrm{pg} / \mathrm{ml}$.

\section{RESULTADOS E DISCUSSÃO}

Foram avaliados dois grupos de amostras compostos por voluntarios saudáveis $(n=65)$, e os casos de câncer de pâncreas $(n=29)$ pareados por sexo $(p=0,1130)$ e idade $(\mathrm{p}=0,1205)$. A mediana de idade dos voluntários sadios e doentes foi 63 e 66 anos, respectivamente. Os pacientes controles apresentavam $81 \%$ e os casos de câncer de pâncreas $65 \%$ de indivíduos do sexo feminino. Todos os soros de pacientes com câncer de pâncreas foram positivos para Gal-9 (mediana $=7462$ ). Sendo observado um aumento significativo $(\mathrm{p}<0,0001)$ em relação aos controles saudáveis (mediana=46.88) (Figura 1).

Figura 1 - Níveis séricos de Gal-9 no soro de voluntários saudáveis e diagnosticados com câncer de pâncreas. $(* * * *=p<0,0001)$. Fonte: próprio autor, 2017.

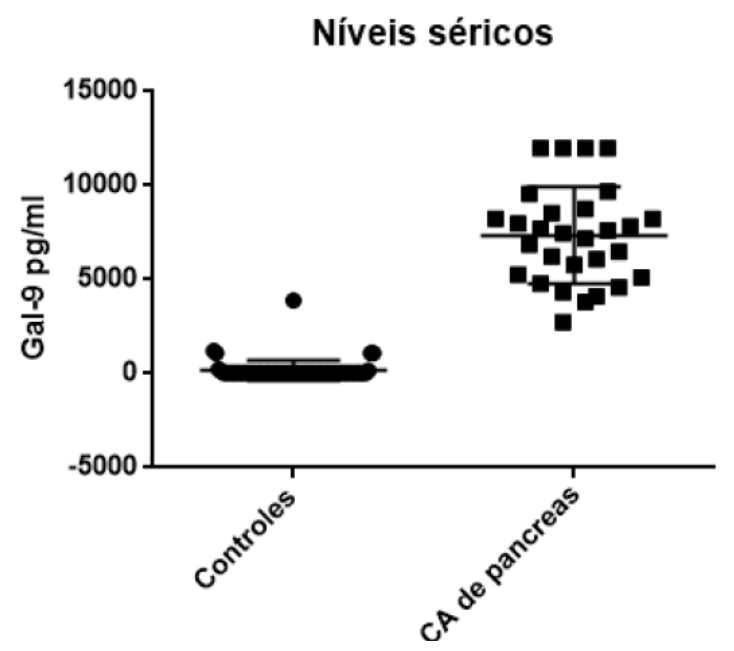

Os níveis de Gal-9 em sobrenadantes de células expostas ficaram abaixo do limite de detecção do kit $\left(<46,875 \mathrm{pg} / \mathrm{ml}\right.$. $\left.\mathrm{R}_{2}=0,8388\right)$. Galectina-9 tem sua expressão alterada em uma variedade de tipos tumorais, incluindo cânceres que afetam o trato gastrointestinal. Viu-se que nos tumores gástrico, adenocarcinoma esofágico e metástases hepáticas provenientes do câncer de pâncreas, essa proteína desempenha papel primordial para supressão do crescimento celular, apoptose e regulação da progressão tumoral (TADOKORO et al., 2017; AKASHI et al., 2017; CHOI et al., 2017 Por isso, acredita-se que as células tumorais podem desempenhar mecanismos adaptativos capazes de inibir a expressão dessa proteína, como observado em tumores hematopoiéticos em associação com outros tipos de galectina (DEMERSA et al., 2009).

\section{CONCLUSÃO}

No presente estudo foi observado que pacientes acometidos pelo câncer de pâncreas apresentam níveis séricos da galectina-9 elevados em relação aos controles saudávies. No entanto, as linhagens celulares avaliadas não representam um bom modelo de avaliação de Gal-9. 


\section{REFERÊNCIAS}

AKASHI, E.; FUJIHARA, S.; MORISHITA, A.; TADOKORO, T.; CHIYO, T.; FUJIKAWA, K.; KOBARA, H.; MORI, H.; IWAMA, H.; OKANO, K.; SUZUKI, Y.; NIKI, T.; HIRASHIMA, M.; MASAKI, T. Effects of galectin-9 on apoptosis, cell cycle and autophagy in human esophageal adenocarcinoma cells. Oncol Rep. 2017 Jul;38(1):506-514.

CÁRDENAS-RODRÍGUEZ, J.; Li, Y.; GALONS, J. P.; CORNNELL, H.; GILLIES, R. J.; PAGEL, M. D.; BAKER, A. F.; Imaging biomarkers to monitor response to the hypoxia-activated prodrug $\mathrm{TH}-302$ in the MiaPaCa2 flank xenograft model. Magnetic Resonance Imaging, 1002-1009. 2012.

CHOI, S. I.; SEO, K. W.; KOOK, M. C.; KIM, C. G.; KIM, Y. W.; CHO, S. J. Prognostic value of tumoral expression of galectin-9 in gastric cancer. Turk J Gastroenterol. 2017 May;28(3):166-170.

CID-ARREGUI, A.; JUAREZ, V.; Perspectives in the treatment of pancreatic adenocarcinoma. World J Gastroenterol, 21(31): 9297-9316. 2015.

DEMERSA, M.; COUILLARDA, J.; GIGLIA-MARIC, G.; MAGNALDOB, T.; STPIERREA, Y. Increased galectin-7 gene expression in lymphoma cells is under the control of DNA methylation.

Biochemical and Biophysical Research Communications, V. 387, 3:(25)425-429. 2009.

HEUSSCHEN, R.; GRIFFIOEN, A. W.; THIJSSEN, V. L.; Galectin-9 in tumor biology: a jack of multiple trades. Biochim Biophys Acta 1836: 177-185, 2013.

IKEMORI, R. Y.; MACHADO, C. M. L.; FURUZAWA, K. M.; NONOGAKI, S.; Osinaga, E.;

UMEZAWA, K.; CARVALHO, M. A.; VERINAUD, L.; CHAMMAS, R.; Galectin-3 Up-Regulation in Hypoxic and Nutrient Deprived Microenvironments Promotes Cell Survival. PLOS one, 2014.

KIM, S. J.; HWANG, J. A.; RO, J. Y.; LEE, Y. S.; CHUN, K. H.; Galectin-7 is epigenetically-regulated tumor suppressor in gastric câncer. Oncotarget, Vol.4, No 9. 2013.

LIU, F. T.; RABINOVICH, G. A.; Galectins as modulators of tumour progression. Nature Reviews Cancer. v. 52005.

LIU, Y.; DU, L.; Role of pancreatic stellate cells and periostin in pancreatic cancer progression. Tumor Biol, 36:3171-3177. 2015.

TADOKORO, T.; FUJIHARA, S.; CHIYO, T.; OURA, L.; SAMUKAWA, E.; YAMANA, Y.; FUJITA, K. Induction of apoptosis by Galectin-9 in liver metastatic cancer cells: In vitro study. Int J Oncol. 2017 Aug;51(2):607-614.

WOLFGANG, C. L.; HERMAN, J. M.; LAHERU, D. A.; KLEIN, A. P.; ERDEK, M. A.; FISHMAN, E. K.; HRUBAN, R. H.; Recent Progress in Pancreatic Cancer. CA Cancer J Clin, 63(5): 318-348. 2013.

XU, Z.; POTHULA, S. P.; WILSON, J. S.; APTE, M. V.; Pancreatic cancer and its stroma: A conspiracy theory. World journal of gastroenterology, 28; 20(32): 1121611229. 2014.

\section{AGRADECIMENTOS}

Núcleo de Pesquisa em Inovação Terapêutica (NUPIT). Coordenação de Aperfeiçoamento de Pessoal de Nível Superior (CAPES). Instituto Nacional de Ciência e Tecnologia para Inovação Farmacêutica (INCT-if). 